\title{
Thyroid adenoma and nasopharyngeal carcinoma with metastasis to cervical lymph nodes is misdiagnosed and treated for thyroid carcinoma: A case report
}

\author{
MIAO ZHANG, HENG WANG, XUEFENG PAN, WENBIN WU and HUI ZHANG \\ Department of Thoracic Surgery, Xuzhou Central Hospital Affiliated \\ to Southeast University, Xuzhou, Jiangsu 221009, P.R. China \\ Received January 14, 2016; Accepted May 20, 2016
}

DOI: $10.3892 / 01.2016 .4612$

\begin{abstract}
Lymph node metastasis of nasopharyngeal carcinoma follows an orderly pattern, and diagnosis of nasopharyngeal carcinoma is often made by lymph node biopsy. In the present study, following neck palpation, ultrasonography and cervical computer tomography, a 52-year-old female patient with thyroid adenoma and enlarged cervical lymph nodes was misdiagnosed as thyroid carcinoma without undergoing preoperative biopsy, followed by unnecessary total thyroidectomy. Systematic CT scan and nasal endoscopic biopsy confirmed the correct diagnosis of primary NPC concurrent with thyroid adenoma. The patient received palliative radiotherapy and L-thyroxine substitution therapy, and was followed up closely via internet-based approaches with life-style intervention, medication consultation and psychological support for improvement of life quality after radiotherapy. In conclusion, primary malignancies with thyroid metastasis must be considered in the differential diagnosis of thyroid tumors with enlarged cervical lymph nodes.
\end{abstract}

\section{Introduction}

The incidence of nasopharyngeal carcinoma (NPC) has become decreased during the past decade (1). The characteristics of cervical lymphatic metastasis in NPC remain to be elucidated (2). NPC has a high propensity of cervical node metastasis, as lymphatic drainage of the nasopharynx is predominantly to cervix (2). Patients with NPC may be asymptomatic even with lymphatic metastasis, and symptoms of NPC mimic that of thyroid carcinoma. Ultrasound in isolation is therefore not reliable for selection of thyroid nodules (3).

Correspondence to: Dr Hui Zhang, Department of Thoracic Surgery, Xuzhou Central Hospital Affiliated to Southeast University, 199 Jiefang South Road, Xuzhou, Jiangsu 221009, P.R. China

E-mail: rfpyfwya03jm@163.com

Key words: nasopharyngeal carcinoma, metastasis, thyroid, misdiagnosis
Thyroid metastasis from NPC is rare and may be correctly diagnosed by fine needle aspiration biopsy (4). Differentiated diagnosis is crucial because therapy regimens are completely different, and simultaneous modulated radiotherapy plus chemotherapy is feasible and effective for patients with advanced NPC (5). Suspicion of multiple primary tumors is essential for patients with head and neck neoplasms.

\section{Case report}

A 52-year-old female was admitted to a local hospital with a painless mass in the anterior neck for 1 year without significant clinical manifestations such as pain, increase in temperature or bleeding. Furthermore, the thyroid function tests were normal. Neck palpation, ultrasonography and cervical computer tomography (CT) showed thyroid nodule and bilateral enlarged cervical lymph nodes, with the largest one approximately $2.0 \mathrm{~cm}$ in diameter (Fig. 1), with a strong suspicion of thyroid carcinoma. A total thyroidectomy and radical neck dissection were performed empirically on the basis of the non-invasive tests. However, the patient suffered from persistent headache and fever for a week, and was transferred to the Xuzhou Central Hospital Affiliated to Southeast University (Jiangsu, China). Following further diagnostic measures, thyroid adenoma and NPC were confirmed by postoperative histopathology (Fig. 2), which was indicated by cranial CT and nasal endoscopic biopsy (Fig. 3). Subsequently, the patient received palliative radiotherapy and L-thyroxine substitution therapy with relatively poor quality of life.

The present study was approved by the Institutional Review Board of the Xuzhou Central Hospital. Informed consent was obtained from the patient.

\section{Discussion}

Lymph node metastasis of NPC follows an orderly pattern, and the most commonly involved regions include retropharyngeal and level II lymph nodes (2). The involvement of cervical and retropharyngeal lymph nodes constitutes a potentially prognostic factor for N1 patients with NPC (2010 UICC/AJCC staging system) (6). Cases of multiple primary malignancies in the same host are often examined, a lymph node biopsy 


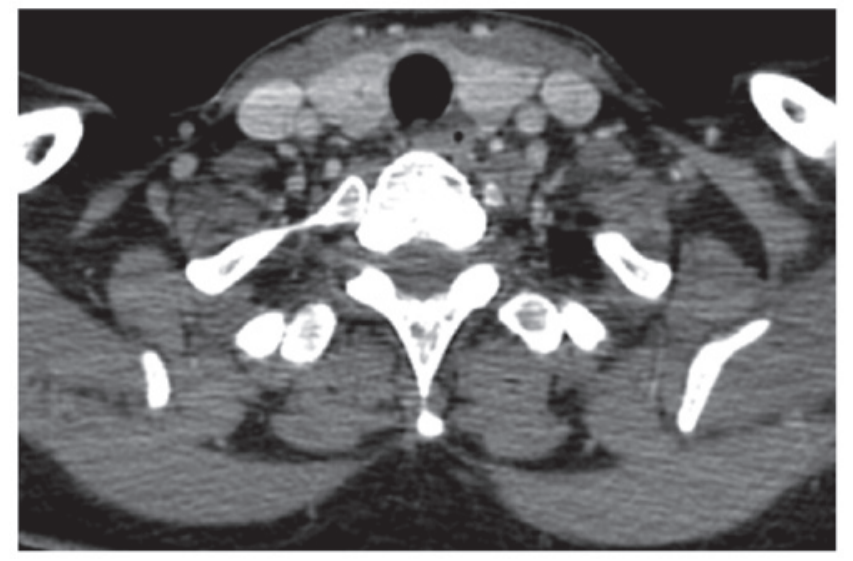

Figure 1. Preoperative computer tomography showed thyroid nodule and enlarged cervical lymph nodes.

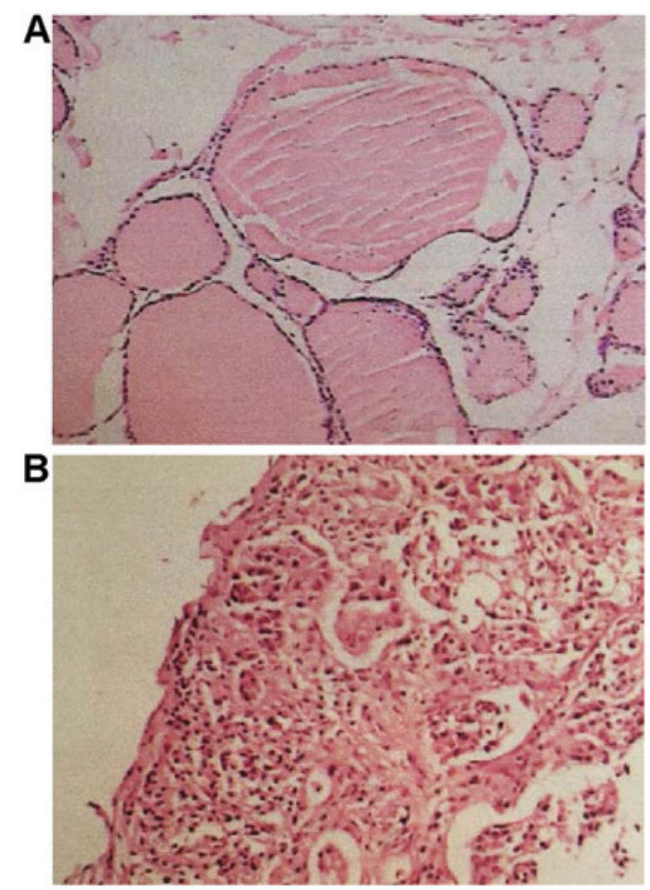

Figure 2. (A) Thyroid adenoma. (B) Metastatic nasopharyngeal carcinoma in dissected cervical lymph nodes confirmed by histopathology (hematoxylin and eosin staining; magnification, $\mathrm{x} 200$ ).

is required for diagnosis of NPC. It has been reported that the most frequent primary malignancies with thyroid metastasis are renal carcinoma, lung cancer and head and neck neoplasms $(7,8)$. Therefore recurrence or progression of non-thyroid malignancy must be considered regardless of time elapsed since the initial diagnosis (7).

In addition to non-invasive procedures such as whole body CT or positron emission tomography, CT ultrasound-guided fine-needle aspiration biopsy of thyroid nodules and lymph nodes, respectively, may be utilized for a concrete differential diagnosis of primary or metastatic neoplasms (9). Prior to treating the patient with any surgical therapy a definite preoperative diagnosis is compulsory. These practices are useful in the avoidance of unnecessary extensive thyroidectomy (as in

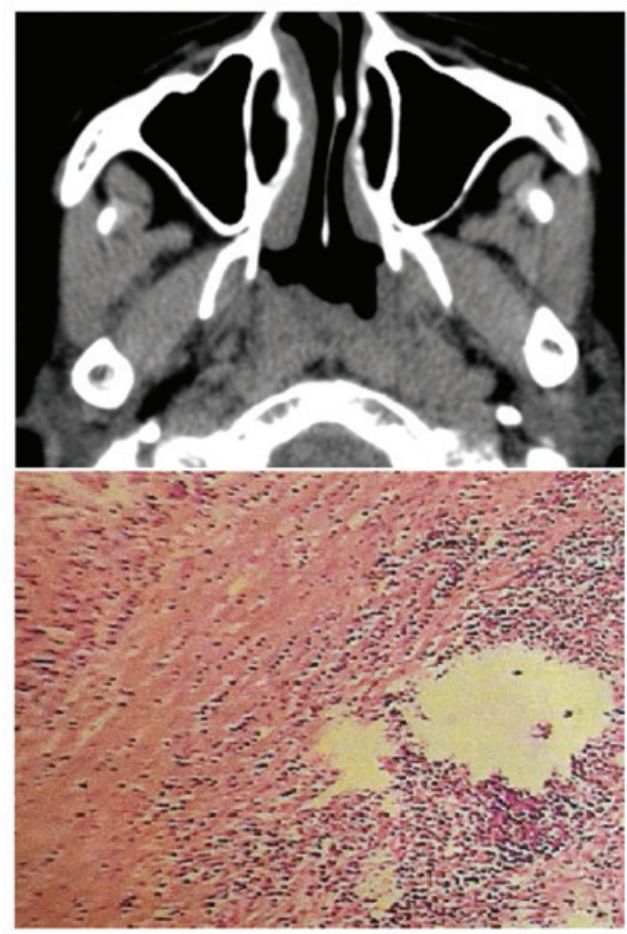

Figure 3. Postoperative additional computer tomography and nasal endoscopic biopsy show the diagnosis of primary nasopharyngeal carcinoma (hematoxylin and eosin staining; magnification, x200).

this particular patient) which would deteriorate the quality of life for patients with NPC. Thus, each case of patient with thyroid nodules and enlarged cervical lymph nodes must be carefully evaluated using histopathology or even immunohistochemistry, and metastatic tumor should be considered in differential diagnosis to avoid misdiagnosis and mistreatment.

In conclusion, metastatic NPC in cervical lymph nodes must be considered and biopsy should be performed separately in the differential diagnosis of thyroid tumors to avoid misdiagnosis and mistreatment.

\section{Acknowledgements}

We appreciate the kind assistance from the staff of the Department of Pathology of Xuzhou Central Hospital. This study was supported by the Speciality Fund for Jiangsu Key Laboratory of Molecular and Translational Cancer Research (no. BM2013007).

\section{References}

1. Chua ML, Wee JT, Hui EP and Chan AT: Nasopharyngeal carcinoma. Lancet 387: 1012-1024, 2016.

2. Ho FC, Tham IW, Earnest A, Lee KM and Lu JJ: Patterns of regional lymph node metastasis of nasopharyngeal carcinoma: A meta-analysis of clinical evidence. BMC Cancer 12: 98, 2012.

3. Remonti LR, Kramer CK, Leitão CB, Pinto LC and Gross JL: Thyroid ultrasound features and risk of carcinoma: A systematic review and meta-analysis of observational studies. Thyroid 25: 538-550, 2015.

4. Chiumento C, Fiorentino A, Castaldo G and Fusco V: A case of thyroid metastasis of nasopharyngeal cancer. Tumori 97: 24e-6e, 2011. 
5. Fan TY, Xing J, Lu J, Liu TH, Xu M, Zhang YJ, Shao Q, Li JB and Yu JM: Phase I/II study of induction chemotherapy plus concurrent chemotherapy and SMART-IMRT-based radiotherapy in locoregionally-advanced nasopharyngeal cancer. Oncol Lett 5: 889-895, 2013.

6. Shi Q, Shen C, Kong L, Wang X, Ding J, Gao Y, Xu T and Hu C: Involvement of both cervical lymph nodes and retropharyngea lymph nodes has prognostic value for N1 patients with nasopharyngeal carcinoma. Radiat Oncol 9: 7, 2014.
7. Chung AY, Tran TB, Brumund KT, Weisman RA and Bouvet M: Metastases to the thyroid: A review of the literature from the last decade. Thyroid 22: 258-268, 2012.

8. Hegerova L, Griebeler ML, Reynolds JP, Henry MR and Gharib H: Metastasis to the thyroid gland: report of a large series from the Mayo Clinic. Am J Clin Oncol 38: 338-342, 2015.

9. Yoon JH, Kwak JY, Kim EK, Moon HJ, Kim MJ, Kim JY, Koo HR and Kim MH: How to approach thyroid nodules with indeterminate cytology. Ann Surg Oncol 17: 2147-2155, 2010. 